\title{
Prevalencia de Cándida y asociación con periodontopatógenos presentes en placa subgingival de pacientes con periodontitis crónica ${ }^{1}$
}

\section{Prevalence of Candida and association with periodontopathogens present in subgingival plaque of chronic periodontitis patients}

\author{
ARDILA MEDINA CM* \\ LÓPEZ GAVIRIA ME** \\ GUZMÁN ZULUAGA IC***
}

Ardila Medina CA, López Gaviria ME, Guzmán Zuluaga IC. Prevalencia de Cándida y asociación con periodontopatógenos presentes en placa subgingival de pacientes con periodontitis crónica. Av Periodon Implantol. 2014; 26, 3: 129-134.

\begin{abstract}
RESUMEN
Introducción: Pocos estudios han descrito la presencia de levaduras en placa subgingival de pacientes con periodontitis crónica desconociéndose las implicaciones que puede tener su presencia sobre el tratamiento periodontal.

Objetivo: El propósito de esta investigación fue evaluar la prevalencia de levaduras en las bolsas periodontales, su influencia sobre los parámetros clínicos y su asociación con otros periodontopatógenos presentes en periodontitis crónica.

Materiales y métodos: Se examinaron los parámetros clínicos (profundidad de bolsa, nivel de inserción, sangrado al sondaje, índice de placa, supuración, movilidad y compromiso de furcación) y la presencia de levaduras en 76 pacientes con periodontitis crónica (promedio de edad, $46 \pm 8$ años) en Medellín, Colombia. Las muestras subgingivales se procesaron mediante cultivo. Para determinar las diferencias de las variables clínicas con la presencia o ausencia de levaduras se utilizaron pruebas de chi cuadrado y U de Mann-Whitney. A través de un coeficiente de correlación no paramétrico (Kendal's $k$ ) se expresó la asociación con otros patógenos periodontales $(p<0,05)$.

Resultados y conclusiones: La prevalencia de levaduras en las bolsas periodontales de pacientes con periodontitis crónica fue de $13,2 \%$. Se encontraron ocho sujetos con C.albicans y dos pacientes con especies de Candida no específicas. Se observó una correlación significativa entre la presencia de levaduras y Prevotella melaninogénica $(\mathrm{p}<0,000)$. El papel que desempeñan las levaduras en la periodontitis crónica no es muy claro y se requieren más estudios para dilucidar el significado clínico de su presencia.
\end{abstract}

PALABRAS CLAVE: Candida, periodontitis crónica, placa subgingival.

\section{SUMIMARY}

Introduction: Few studies have described the presence of subgingival plaque of patients with chronic periodontitis and were unaware of the implications that may have its presence in the periodontal treatment.

Objective:The purpose of this research was to assess the prevalence of yeasts in periodontal pockets, their influence on clinical parameters and their association with other periodontopathogens in chronic periodontitis.

Investigación financiada por la Facultad Nacional de Salud Pública y el Grupo de Epidemiología de la Universidad de Antioquía.

* Profesor Asociado Facultad de Odontología Universidad de Antioquía. Candidato a PhD en Epidemiología Universidad de Antioquía. Miembro Junta Directiva Asociación Colombiana de Periodoncia y Oseointegración.

** Higienista Oral Facultad de Odontología Universidad de Antioquía.

*** Periodoncista. Universidad de Chile. Profesora Facultad de Odontología Universidad de Antioquía. 
Materials and methods: We reviewed the clinical parameters (pocket depth, clinical attatchment level, bleeding on probing, plaque index, suppuration, mobility and furcation involvement) and the presence of yeast in 76 patients with chronic periodontitis (mean age, $46 \pm 8$ years) in Medellin, Colombia. Subgingival samples were processed by culture. To determine the differences in clinical variables with the presence or absence of yeasts were used chi square and Mann-Whitney tests. Through a non-parametric correlation coefficient (Kendal's k) expressed the association with other periodontal pathogens $(\mathrm{p}<0.05)$.

Results and conclusions: The prevalence of yeasts in periodontal pockets of patients with chronic periodontitis was $13.2 \%$. There were eight subjects with $C$. albicans and two patients with non-specific species of Candida. There was a significant correlation between the presence of yeast and Prevotella melaninogenica $(\mathrm{p}<0.000)$. The role of yeasts in chronic periodontitis is not clear and further studies are needed to demonstrate the clinical significance of their presence..

KEY WORDS: Candida, chronic periodontitis, subgingival plaque.

Fecha de recepción: 8 de septiembre de 2009.

Fecha de aceptación: 15 de septiembre de 2009.

\section{INTRODUCCIÓN}

La periodontitis es una enfermedad infecciosa inflamatoria que se caracteriza por la destrucción de los tejidos de soporte (1). La periodontitis crónica, la forma más frecuente de periodontitis, está asociada a una compleja y diversa variedad de microorganismos constituidos por bacterias gram-positivas y gram-negativas, organismos anaerobios y facultativos $(2,3)$. Diferentes estudios también han documentado la presencia de levaduras en las bolsas periodontales sugiriendo una función potencial de estos organismos en la patogénesis de la periodontitis (4). La presencia de levaduras en diferentes regiones del cuerpo humano incluyendo la cavidad bucal se ha asociado a salud y enfermedad $(5,6)$. Las levaduras son patógenos oportunistas que causan enfermedad en huéspedes comprometidos por procesos patológicos locales o sistémicos (6). Se ha sugerido que el uso de antibióticos de amplio espectro puede desencadenar sobreinfecciones con levaduras en la bolsa periodontal como resultado del disturbio que causan en la homeostasis de la microflora habitual (7). En la cavidad bucal, las levaduras colonizan lengua, paladar, mucosa oral y saliva (8). Específicamente, la Candida Albicans se ha encontrado en gran cantidad en bolsas periodontales $(7,1-26,9 \%)$ de pacientes con periodontitis crónica (2-4, 9-11). Algunos estudios sugieren que la colonización subgingival por levaduras puede estar favorecida en sujetos con periodontitis crónica $(3,5)$, sin embargo, ha recibido poca importancia el papel de las levaduras en esta clase de periodontitis a pesar del carácter polimicrobiano de la enfermedad periodontal, $(2,3,5)$. Los objetivos de esta investigación fueron evaluar la prevalencia de levaduras en bolsas periodontales de pacientes con periodontitis crónica y estudiar su asociación con los periodontopatógenos presentes en placa subgingival. Se estudiaron también las características clínicas de los sujetos con levaduras en placa subgingival.

\section{MATERIALES Y MÉTODOS}

\section{Sujetos}

Se invitaron a participar en este estudio 76 sujetos sistémicamente sanos (45 mujeres y 31 hombres) que asistieron a las clínicas odontológicas de la Facultad de Odontología de la Universidad de Antioquía entre octubre de 2008 y marzo de 2009. Cada participante firmó un consentimiento informado. El Comité de Ética de la Sede de Investigación Universitaria de la Universidad de Antioquía aprobó el diseño del estudio teniendo en cuenta la Declaración de Helsinki sobre experimentación que involucra seres humanos. Se consideraron candidatos para participar en la presente investigación pacientes con diagnóstico de periodontitis crónica. Los criterios de exclusión fueron embarazo, lactancia, presencia de diabetes o cualquier enfermedad sistémica que alterara el curso de la enfermedad periodontal, terapia periodontal en el último año, y utilización de antimicrobianos o antiinflamatorios no esteroides en los seis meses previos al examen clínico y a la toma de muestras microbiológicas. 


\section{Evaluación clínica}

A cada paciente se le realizó una historia clínica, además de un examen clínico y radiográfico completo. Uno de los autores $(\mathrm{CA})$ realizó todos los exámenes clínicos. Se registraron los siguientes parámetros clínicos: profundidad de sondaje, nivel de inserción clínica, sangrado al sondaje, índice de placa, supuración, movilidad y compromiso de furcación. La profundidad de sondaje y el nivel de inserción clínica se midieron en seis sitios (mesiobucal, bucal, distobucal, distolingual, lingual y mesiolingual) usando una sonda periodontal calibrada (UNC-15, Hu-Friedy, Chicago, IL). El diagnóstico de periodontitis crónica se realizó según los criterios de la Academia Americana de Periodoncia (AAP) (12).

\section{Muestreo microbiológico}

Se tomaron muestras microbiológicas de los pacientes en sitios con una profundidad de sondaje mayor a $5 \mathrm{~mm}$. Para el muestreo se seleccionaron las seis bolsas periodontales más profundas. Después de aislar la zona con algodón y remover la placa supragingival con cureta se insertaron dos conos de papel estéril en cada bolsa periodontal durante 20 segundos. Las muestras de cada paciente se depositaron en $2 \mathrm{~mL}$ de medio de transporte y se llevaron al laboratorio de microbiología de la Facultad de Odontología de la Universidad de Antioquía para procesarlas dentro de las dos horas siguientes. A partir de las muestras subgingivales se hicieron cultivos microbiológicos en agar TSBV39 y en agar Brucella suplementado con sangre de cordero al $5 \%$, hemina y menadiona (13). Las cajas de agar TSBV se incubaron en una atmósfera al $5 \%$ de $\mathrm{CO}_{2}$ entre 3 y 5 días (permite recuperación de Aggregatibacter actinomycetemcomitans y también el crecimiento de levaduras). Las cajas de agar Brucella se incubaron en anaerobiosis por siete días para la recuperación de los demás periodontopatógenos. La identificación se hizo teniendo en cuenta características morfológicas de las colonias y pruebas bioquímicas adicionales (14). Para identificar las levaduras se subcultivó la colonia presuntiva en agar TSBV al agar sangre evaluándose el aspecto de la colonia y la morfología microscópica correspondiente a blastoconidias en la preparación con azul de lactofenol (15).

\section{Análisis estadístico}

Se realizó inicialmente un análisis estadístico descriptivo univariado. Posteriormente mediante pruebas de chi cuadrado se realizó un análisis bivariado entre los perfiles bacterianos y variables sociodemográficas (edad y género) versus la presencia o ausencia de levaduras. Mediante la prueba U de Mann-Whitney se determinaron las diferencias en la profundidad de bolsa y nivel de inserción clínica entre hombres y mujeres y la presencia o ausencia de levaduras. La asociación con otros patógenos periodontales se expresó a través de un coeficiente de correlación no paramétrico (Kendal's $k$ ). El valor de significación para todas las pruebas se estableció como $p=0,05$. Se utilizó un programa estadístico para todos los análisis (SPSS, versión 15, Chicago, IL).

\section{RESULTADOS}

Se estudiaron 31 hombres (41\%) y 45 mujeres (59\%) con periodontitis crónica avanzada. La tabla 1 presenta las características clínicas de los pacientes evaluados. Los hombres y mujeres examinados mostraron parámetros clínicos diferentes. Se encontró mayor pérdida de inserción en los hombres y también porcentajes más elevados de placa, supuración, movilidad, y compromiso de furcación comparado con las mujeres (diferencias estadísticamente significativas). Además se observó un mayor porcentaje de hombres fumadores $(p<0,001)$.

La tabla 2 presenta las características clínicas de los pacientes con 0 sin presencia de levaduras en placa subgingival. Los hombres con presencia de levaduras presentaron mayor pérdida de inserción clínica comparado con las mujeres con presencia de levaduras, diferencias que fueron estadísticamente significativas $(3,81 \pm 1,7 \mathrm{~mm}$ versus $2,94 \pm 1,2 \mathrm{~mm} ; \mathrm{p}=0,008)$. Igualmente, los hombres con presencia de levaduras también fumaban en mayor porcentaje $(30 \%$ hombres versus $20 \%$ mujeres; $p=0,002$ ).

La prevalencia de levaduras en las bolsas periodontales de pacientes con periodontitis crónica fue de $13,2 \%$ (10/76). En el grupo de sujetos con levaduras, las mujeres presentaron mayor cantidad de este microorganismo $(15,8 \%, 7 / 45)$ comparado con los hombres $(9,8 \%, 3 / 31)$, diferencias que fueron estadísticamente significativas $(p<0,000)$. Se encontraron ocho sujetos con C. albicans y dos pacientes con especies de Candida no específicas.

Se encontró una correlación débil pero significativa entre la presencia de levaduras y Prevotella melaninogénica $(\mathrm{r}=0,181, \mathrm{p}<0,001)$. 


\section{TABLA 1.- CARACTERÍSTICASS CLÍNICAS DE 76 PACIENTES CON PERIODONTITIS CRÓNICA}

\begin{tabular}{|l|c|c|c|}
\hline & Hombres & Mujeres & Valor p \\
\hline Edad & $47 \pm 9$ años & $45 \pm 8$ años & 0,5 \\
\hline Profundidad bolsa & $3,1 \pm 1,3 \mathrm{~mm}$ & $2,9 \pm 1,2 \mathrm{~mm}$ & 0,5 \\
\hline Nivel inserción & $3,8 \pm 1,2 \mathrm{~mm}$ & $3,3 \pm 1,6 \mathrm{~mm}$ & $<0,001$ \\
\hline Sangrado & $74 \%$ & $73 \%$ & 0,5 \\
\hline Placa & $56 \%$ & $48 \%$ & $<0,001$ \\
\hline Supuración & $17 \%$ & $13 \%$ & 0,006 \\
\hline Movilidad & $25 \%$ & $20 \%$ & 0,014 \\
\hline Compromiso de furcaciones & $19 \%$ & $16 \%$ & 0,05 \\
\hline Hábito de fumar & $29 \%$ & $18 \%$ & $<0,001$ \\
\hline$\pm:$ Desviación estándar & & & \\
\hline
\end{tabular}

\section{TABLA 2.- CARACTERÍSTICAS CLÍNICASS DE LOS PACIENTES CON O SIN PRESENCIA DE LEVADURAS EN PLACA SUBGINGIVAL}

\begin{tabular}{|l|c|c|c|}
\hline & $\begin{array}{c}\text { Presencia } \\
\text { de levaduras }\end{array}$ & $\begin{array}{c}\text { Ausencia } \\
\text { de levaduras }\end{array}$ & Valor p \\
\hline Hombres & $30 \%$ & $42 \%$ & $<0,001$ \\
\hline Mujeres & $70 \%$ & $58 \%$ & $<0,001$ \\
\hline Profundidad bolsa & $2,99 \pm 1,2 \mathrm{~mm}$ & $2,94 \pm 1,2 \mathrm{~mm}$ & 0,5 \\
\hline Nivel inserción & $3,8 \pm 1,7 \mathrm{~mm}$ & $3,5 \pm 1,8 \mathrm{~mm}$ & 0,008 \\
\hline Sangrado & $69 \%$ & $74 \%$ & 0,1 \\
\hline Placa & $46 \%$ & $52 \%$ & 0,09 \\
\hline Supuración & $14 \%$ & $13 \%$ & 0,6 \\
\hline Movilidad & $20 \%$ & $21 \%$ & 0,8 \\
\hline Compromiso de furcaciones & $15 \%$ & $16 \%$ & 0,8 \\
\hline Hábito de fumar & $30 \%$ & $21 \%$ & 0,002 \\
\hline$\pm:$ Desviación estándar & & & \\
\hline
\end{tabular}

\section{DISCUSIÓN}

En este estudio se investigó la prevalencia de levaduras en 76 pacientes con periodontitis crónica, estudiando además las características clínicas de los su- jetos que presentaron levaduras en placa subgingival y la asociación de estos microorganismos con otros periodontopatógenos. La prevalencia de levaduras en esta investigación (13,2\%) fue similar a la encontrada por Slots y colaboradores (16) quienes 
registraron $13-14 \%$ de levaduras en bolsas periodontales de pacientes con periodontitis refractaria. Como en el presente estudio C. albicans fue la levadura más frecuente. Estos resultados son corroborados por otros estudios que también han encontrado una proporción mayor de $C$. albicans en pacientes con periodontitis crónica $(2,3)$.

En esta investigación se presentó una mayor frecuencia de levaduras en las mujeres comparado con los hombres (Tabla 2), como también fue demostrado por Slots y colaboradores (16). Este incremento en la presencia de levaduras en las mujeres puede relacionarse con la mayor frecuencia de periodontitis refractarias en el sexo femenino (16).

Al comparar los parámetros clínicos de los pacientes con presencia de levaduras versus ausencia de levaduras se observaron diferencias estadísticamente significativas solamente en el nivel de inserción (Tabla 2). También se encontró un mayor porcentaje de fumadores en el grupo de pacientes con presencia de levaduras en placa subgingival (Tabla 2). El hábito de fumar se ha asociado con mayor pérdida de inserción en pacientes con periodontitis (17) como se observó en el presente estudio, sin embargo, nuestros datos no pueden confirmar una asociación entre hábito de fumar y presencia de levaduras en bolsas periodontales. Se requieren más estudios que evalúen esta asociación.

Muy pocas investigaciones han estudiado la asociación entre levaduras presentes en placa subgingival de pacientes con periodontitis crónica y otros periodontopatógenos. En esta investigación se encontró una correlación débil pero estadísticamente significativa entre levaduras y P. melaninogénica. Igualmente, Hidenobu y colaboradores (18) encontraron también una asociación entre estos dos microorganismos en la placa bacteriana de sujetos con halitosis. Es recomendable realizar estudios longitudinales con el fin de evaluar estas posibles asociaciones.

C. albicans puede cumplir una función importante en la infraestructura de la placa subgingival y en su adherencia a los tejidos periodontales (5). Jarvensivu y colaboradores (5) encontraron que $C$. albicans puede participar en la evasión inmune de la placa en las infecciones periodontales y puede penetrar el epitelio de la bolsa periodontal provocando inflamación destructiva de los tejidos. El papel que desempeña C. albicans en la periodontitis crónica no es muy claro y se requieren más estudios para demostrar el significado clínico de sus hallazgos. Los resultados del presente trabajo soportan que C. albicans puede ser parte del proceso de la periodontitis crónica.

\section{CONCLUSIONES}

Es poco claro el papel potencial que cumple la presencia de levaduras en la placa subgingival de pacientes con periodontitis crónica. Así mismo, se desconoce la función que desempeña este microorganismo en la exacerbación de las condiciones clínicas de la enfermedad periodontal y sus posibles implicaciones en el tratamiento.

\section{BIBLIOGRAFÍA}

1. Kornman, KS, Page RC,Tonetti MS. The host response to the microbial challenge in periodontitis: assembling the players. Periodontology 2000 1997;14:33-53.

2. Reynaud AH, Nygaard-Østby B, Bøygard G-K, Eribe ER, Olsen I, Gjermo P. Yeasts in periodontal pockets. J Clin Periodontol 2001;28:860-4.

3. Urzúa B, Hermosilla G, Gamonal J, Morales-Bozo I, Canals $\mathrm{M}$, Barahona S, et al. Yeast diversity in the oral microbiota of subjects with periodontitis: Candida albicans and Candida dubliniensis colonize the periodontal pockets. Med Mycol; 2008:46:783-93.

4. Papapanou PN. Population studies of microbial ecology in periodontal health and disease. Ann Periodont 2002;7: 54-61.

5. Jarvensivu A, Hietanen J, Rautemaa R, Sorsa T, Richardson M. Candida yeasts in chronic periodontitis tissues and subgingival microbial biofilms in vivo. Oral Dis 2004; 10 : 106-12.

6. McCullough MJ, Ross BC, Reade PC. Candida albicans: a review of its history, taxonomy, epidemiology, virulence attributes, and methods of strain differentiation. Int J Oral Maxillofac Surg 1996;25:136-44.

7. Helovuo H, Hakkarainen $\mathrm{K}$, Paunio $\mathrm{K}$. Changes in the prevalence of subgingival enteric rods, staphylococci and yeasts after treatment with penicillin and erythromycin. Oral Microbiol Immunol 1993;8:75-9.

8. Hannula J, Dogan B, Slots J, Okte E, Asikainen S. Subgingival strains of Candida albicans in relation to 
geographical origin and occurrence of periodontal pathogenic bacteria. Oral Microbiol Immunol 2001;16: 113-18.

9. Rams TE, Flynn MJ, Slots J. Subgingival microbial associations in severe human periodontitis. Clin Inf Dis 1997;25 (Suppl. 2):S224-6.

10. Dahlén G, Wikstrom M. Occurrence of enteric rods, staphylococci and Candida in subgingival samples. Oral Microbiol Immunol 1995;10:42-6.

11. Slots J, Rams TE, Listgarten MA. Yeasts, enteric rods and pseudomonads in the subgingival flora of severe adult periodontitis. Oral Microbiol Immunol 1988;3:47-52.

12. Armitage GC. Development of a classification system for periodontal diseases and conditions. Ann Periodontol 1999;4:1-6.

13. Slots J. Selective medium for isolation of Actinobacillus actinomycetemcomitans. J Clin Microbiol 1982;15:606-9.

14. Slots J. Rapid identification of important periodontal microorganisms by cultivation. Oral Microbiol Immunol 1986; 1:48-55.
15. Koneman EW. Diagnóstico microbiológico. Texto y Atlas color. 5a ed. Madrid: Editorial Médica Panamericana; 1999.

16. Slots J, Feik D, Rams TE. Age and sex relationships of superinfecting microorganisms in periodontitis patients. Oral Microbiol Immunol 1990;5:305-8.

17. Phipps KR, Chan BK, Jennings-Holt M, Geurs NC, Reddy MS, Lewis CE, et al. Periodontal health of older men: the MrOS dental study. Gerodontology 2009;26:122-9.

18. Senpuku H, Tada A, Yamaga T, Hanada N, Miyazaki H. Relationship between volatile sulphide compounds concentration and oral bacteria species detection in the elderly. Int Dent J 2004;54:149-53.

\section{CORRESPONDENCIA}

\section{Carlos Martín Ardila M.}

Facultad de Odontología. Universidad de Antioquía Calle $64 \mathrm{~N}^{\circ}$ 52-59

Medellín, Antioquía, Colombia.

Correo electrónico: martinardila@gmail.com 